\title{
ANALYSIS OF THE CHANDLER PERIOD OF POLAR COORDINATES CALCULATED BY THE ORLOV METHOD
}

\author{
E. PROVERBIO \\ International Astronomical Station, Carloforte-Cagliari, Italy \\ and \\ F. CARTA and F. MAZZOLENI \\ Astronomical Observatory, Brera-Milano, Italy
}

\begin{abstract}
The coordinates $x$ and $y$ of the pole related to mean pole of the epoch in the period 1900-1962 for the ILS station were calculated utilising the method proposed by Orlov (1955). The values of the latitudes are those reduced in the homogeneous system of Carnera-Cecchini by Proverbio et al. (1969). The values of the amplitudes and periods of the polar motion were calculated. The comparison of the data seems to confirm a correlation between the amplitudes and periods of Chandler motion, that is the validity of the time-variable model suggested by Melchior (1949) and other authors.
\end{abstract}

\section{Introduction}

The analysis of latitude variations has led, as is known, to the emphasizing of periodic and irregular variations of polar and local origin.

The analysis of periodic variations could, however, be relatively simplified by first eliminating the existence of long-term secular or irregular variations.

In 1955, Orlov proposed a method of calculating the polar coordinates, based on the equations of polar motion given by Helmert and in which the results in practice refer to the mean pole of the epoch. The procedure used by Orlov is based on the representation of the instantaneous coordinates of the pole by means of the following relationships:

$$
\begin{aligned}
& x=A \cos \left(36^{\circ} t+a\right)+C \cos \left(30^{\circ} t+c\right), \\
& y=B \cos \left(36^{\circ} t+b\right)+C \cos \left(30^{\circ} t+c\right) .
\end{aligned}
$$

The only hypotheses on which the method is based take into account the fact that the movement of the pole of inertia, mainly caused by meteorological phenomena, may be considered known and that during brief intervals of time, of the order of a Chandler period, the amplitude $C$ and the phase $c$ of the Chandler components may be considered, to a sufficiently good approximation as constant.

\section{Amplitude and Period of the Chandler Component}

In 1949, Melchior suggested a mechanical model of the Earth consisting of a uniform nucleus and a mantle with different rigidity, so as to interpret the variations of the period and the amplitude of the free movement found by several workers (Kimura, 1918; Labrouste,1946; Nicolini, 1948), as owing to the superposition of two different periods. 
Recently however, Nesterov and Rykhlova (1970), by analyzing the observations made at Pulkovo, Greenwich and Washington and by determining the length of the Chandler period by a method equivalent to the method of maximum likelihood (Arato et al., 1962) conclude that no relation is present between the length of the Chandler period, its amplitude, and the amplitude of the annual motion. This result would therefore be a confirmation of the so-called damped model of polar motion based on the theory of a random excitation of the Earth.

By using the values of the quantities $C$ and $\left(c+\lambda_{i}\right)$ calculated for successive periods of $1.2 \mathrm{yr}$ using the reduction method proposed by Orlov (1955), it becomes possible to determine the amplitude and the periods characteristic of each interval. For the calculation of these intervals, considering in fact the phase $\left(c+\lambda_{i}\right)=\alpha_{1}+\alpha_{2} t$ variable as a function of time $t$, we can write

$$
P_{t}=P\left(1+(P / 2 \pi) \alpha_{2}\right)^{-1}
$$

where $P=1.2 \mathrm{yr}$ is the average value of the Chandler period considered, while

$$
\alpha_{2}=\frac{\Delta\left(c+\lambda_{i}\right)}{\Delta t} \quad(\Delta t=P)
$$

is the observed variation of the phase $\left(c+\lambda_{i}\right)$ in the time interval $\Delta t=P=1.2 \mathrm{yr}$.

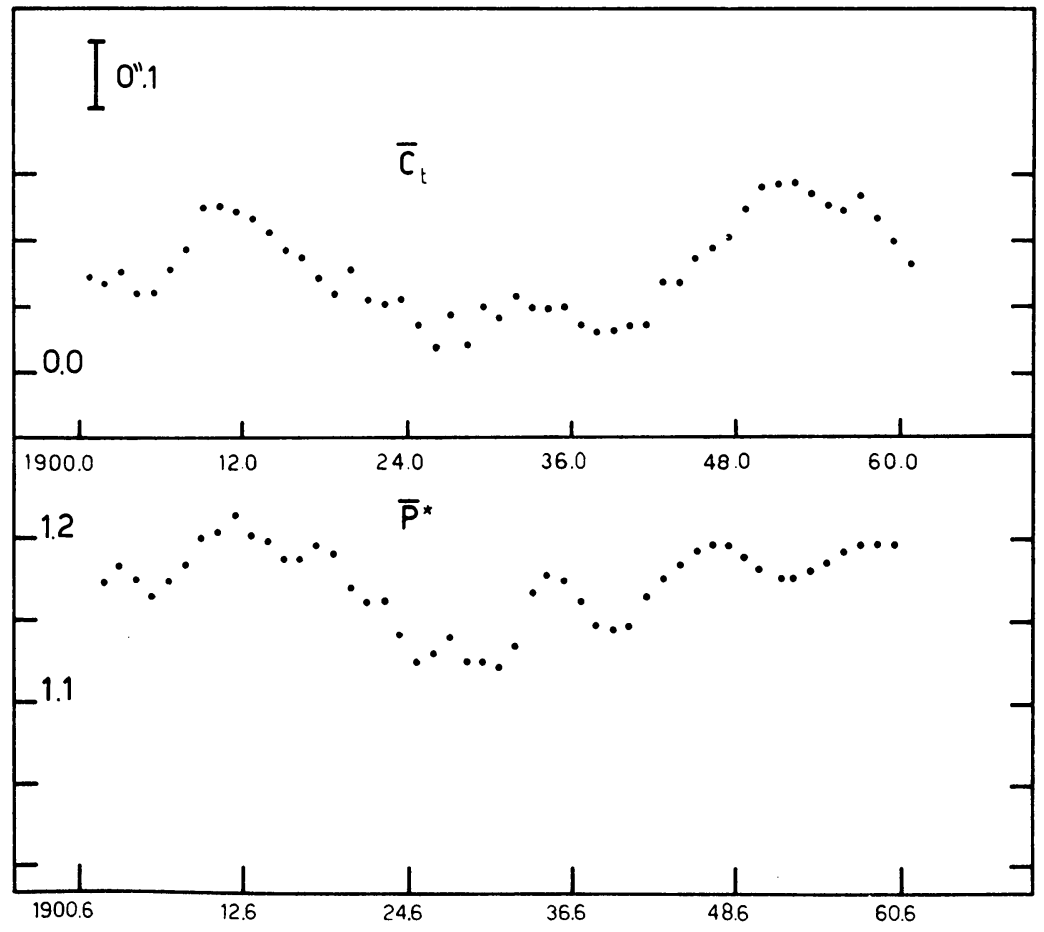

Fig. 1. 
Using these quantities and referring to relationship (2), in which, as has been said, $\alpha_{2}$ represents the phase variation observed for every interval of $1.2 \mathrm{yr}$, the values of the period observed $P_{t}$ for each station $n$ were determined.

In Figure 1 we find the diagram of the average amplitudes

$$
\bar{C}_{t}=\frac{1}{n} \sum_{1}^{n} C_{t n}
$$

relative to $n$ stations of the ILS available in the various interval together with the smoothed values $\bar{P}_{t}^{*}$ of the quantities

$$
\bar{P}_{t}=\frac{1}{n} \sum_{1}^{n} P_{t n} .
$$

The trend of these quantities seems to confirm a correlation between the amplitude and period of the Chandler motion and thus a confirmation of the time-variable model suggested by Melchior and other authors.

Details are described in Proverbio et al. (1971).

\section{References}

Arato, M., Kolmogorov, A. N., and Sinai, Ya. G.: 1962, Dokl. Akad. Nauk 146, 747.

Kimura, H.: 1918, Monthly Notices Roy. Astron. Soc. 78, 163.

Melchior, P.: 1949, Comm. Obs. Roy. Belgique, No. 10.

Melchior, P.: 1951, Comm. Obs. Roy. Belgique, No. 28.

Melchior, P.: 1952, Comm. Obs. Roy. Belgique, No. 36.

Nesterov, V. V., and Rykhlova, L. V.: 1970, Astron. J. 47, 1426.

Nicolini, T.: 1948, Atti Acad. Naz. Lincei, VIII (II).

Orlov, A. Ia.: 1946, Compt. Rend. Acad. Sci. U.S.S.R. 51, 509.

Orlov, A. Ia.: 1955, Trudy Polt. Gravim. Obs. V, 17.

Proverbio, E., Carta, F., and Mazzoleni, F.: 1969, Contr. Oss. Astron. Milano, No. 319.

Proverbio, E., Carta, F., and Mazzoleni, F.: 1971, Mem. SAIt, XLII, 497. 